\title{
Hemodialysis Care by Nurses: Exploration of Performance Factors at Four Nephrology Units in Rwanda
}

\author{
Marie Jeanne Tuyisenge ${ }^{1 *}$, Providence Umuziga ${ }^{1}$, Emeline Cishahayo Umutoni ${ }^{1}$, Violette Dushimiyimana ${ }^{1}$, Marie \\ Josee Mwiseneza ${ }^{1}$, Innocent Hakizimana ${ }^{2}$, Oluyinka Adejumo ${ }^{1,3}$, Geldine Chironda ${ }^{1,3}$ \\ ${ }^{1}$ School of Nursing and Midwifery, University of Rwanda, College of Medicine and Health Sciences, Kigali, Rwanda \\ ${ }^{2}$ Centre for Language Enhancement, University of Rwanda, Kigali, Rwanda \\ ${ }^{3}$ Rory Meyers College of Nursing, New York University, New York, USA
}

*Corresponding author: Marie Jeanne Tuyisenge. School of Nursing and Midwifery, College of Medicine and Health Sciences, University of Rwanda, Remera Campus, 11 KG 47, Kigali, Rwanda. Email: mjsenge@gmail.com

\begin{abstract}
Background

Hemodialysis is the most standard method of Renal Replacement Therapy (RRT) used in Rwanda. Nephrology nursing is a new speciality, and little is known about the nurses' work performance while caring for patients under going hemodialysis.

Objective

To explore factors influencing nurse's work performance in the care of hemodialysis patients in four nephrology units in Rwanda.

Methods

A qualitative descriptive design used a purposive sampling strategy to recruit 12 registered nurses working on the hemodialysis unit. Data were collected through interviews using a semistructured guide. Data analysis used a thematic approach with six phases.

Results

Two themes and eight subthemes were revealed. Facilitating factors to increase work performance included the nurses' education and speciality training, good working environment, good interpersonal relationships among staff, and effective management and leadership. Barriers included, self-perceived knowledge and skills insufficiencies, moral distress, logistical concerns, and poor working conditions.

Conclusion

The findings revealed specific facilitating factors and barriers to nurses' work performance. Nurses at these study sites, in conjunction with faculty in the Master's Nephrology Track at the University of Rwanda, could become change agents and begin to increase facilitating factors and decrease barriers. Continual improvement in facilitating factors is needed to keep nurses performing well.
\end{abstract}

Rwanda J Med Health Sci 2019;2(2):178-184.

Keywords: Nephrology, hemodialysis, nurses, work performance

\section{Background}

Chronic Kidney Disease (CKD) has become a significant health problem worldwide, with a noticeable burden in Low and Middle-Income Countries (LMIC).[1] This condition has been aggravated by an increased prevalence of Type 2 diabetes mellitus (T2DM), hypertension and HIV, leading to increased morbidity and mortality. [1] Moreover, CKD is affecting younger age groups, especially in developing countries like Rwanda, where it affects not only the individual but also the community and country.[1]

As kidney disease progresses, it results in End-Stage Renal Disease (ESRD) and requires regular hemodialysis (HD) treatment. ESRD is defined as a glomerular filtration rate (GFR) of less than $15 \mathrm{~mL}$ per minute per $1.73 \mathrm{~m} 2$ body surface area.[2] ESRD is one of the complications of T2DM, a chronic condition with a prevalence of 336 million in 2012 and expected to rise to 522 million by 2030,[3] indicating a greater need for HD treatment and more intervention by nurses over the next decade.

An increase of the prevalence of ESRD patients necessitates better dialysis management for improved patient outcomes. [4] While kidney transplantation is the best treatment, resource constraints and the shortage of kidney donors limits this option in Africa. HD is also expensive, but a viable alternative.[5] In Rwanda, HD started at one facility a decade ago and in 2014 the government expanded availability due to high mortality 
and lack of Renal Replacement Therapy (RRT). There are now four HD facilities; three in Kigali City, and one in the rural southern province.[6]

Healthcare providers require specific knowledge and a skill set to manage the high-technology equipment, machines, and needles of HD treatment.[7] Nurses need the knowledge and expert skills to meet patients' needs. [8] Since HD is a relatively new treatment, little is known about the work performance of nurses administering this treatment. Several studies address factors related to work performance by nurses, [9-11] and a recent review,[10] reported on factors influencing nurses working conditions in hospitals. However, there is no study published on the factors related to nurses' work performance when administering HD treatment in this expanding speciality field in Rwanda. This study aimed to explore the factors influencing nurse's work performance in the care of HD patients at four nephrology units in Rwanda.

\section{Methods}

\section{Design}

A descriptive qualitative design was used to explore the factors influencing nurses' work performance while caring for HD patients. This study was conducted at four HD units; the University Teaching Hospital of Kigali, the University Teaching Hospital of Butare, King Faisal Hospital, and the African Health Network. Two HD units were at public health institutions and two at private HD centres, though the centres received ESRD patients from all areas of Rwanda. Data were collected from 13 February to 13 April 2017.

\section{Participants' recruitment}

Twenty-one registered nurses were working in the HD units during the study period, though the sample consisted of 12 nurses, based on the study objectives and guided by data saturation. A purposive sampling method was used to target registered nurses who had work experience in HD settings.

\section{Measures}

A semi-structured interview guide was developed based on an in-depth literature review and support from nephrology experts in the nephrology speciality. The guide included open-ended questions and probing questions to facilitate nurses to fully express their thoughts and feelings about factors influencing their work performance in the care of HD patients. The interview guide was piloted on four nurses from different HD centres for content validity and after that adapted with minor changes for clarity.

\section{Data collection}

Prior to data collection, appointments were set-up with participants to select the most appropriate time to conduct the study. Upon convening, a quiet room was selected for the discussion. The investigator provided detailed information about the study, obtained permission through a signed consent, and recorded the interview. The participants were interviewed in English or French, depending on their preference. The interviews were later translated verbatim into transcripts in English. Participants were aware their personal information would be confidential and responses coded for anonymity.

\section{Trustworthiness}

Trustworthiness of the qualitative data was identified through the five following criterion.[12] Credibility provided internal validity with recorded transcripts, and direct quotes resulted in truth-value. Dependability evaluates the reliability, and similar findings would be likely with other registered nurses working in HD patients in Rwanda. Confirmability was a reflection of the interpretation of the participants' voices. Transferability evaluates the external validity and may be possible to other contexts in Rwanda. Authenticity was obtained through the direct quotes of the lived experiences of the nurses working on the dialysis units day and night.

\section{Data analysis}

Data were analysed using a Thematic Analysis as outlined by Braun and Clarke and included six phases. [13] Phase one involved familiarisation of the data, with reading and rereading the transcripts and the field notes, searching for meanings and patterns in order to understand the whole content. In phase two the data were coded using abbreviations, written notes, and two different colors specified possible patterns. The yellow colour indicated facilitating factors (FF), and the green colour indicated barriers (B). In addition, codes were numbered, as there were many. In phase three the search went beyond the different codes looking for possible themes. Then all the relevant coded data was collated into specific themes. Phase four involved reviewing and refining the themes, so they were meaningful and exclusive. Some initial codes were combined to form the main themes, while others formed sub-themes. In phase five two main themes emerged: "Facilitating factors" and "Barriers" to nurses' work performance. Phase six displayed the findings in table 1 .

\section{Ethical considerations}

Ethical approval from the University of Rwanda, College of Medicine and Health Sciences, Institutional Review Board and permission from the associated hospital's review boards was obtained prior to data collection.

\section{Results}

Twelve registered nurses were interviewed using a semi-structured questionnaire. Most participants were females, aged between 20 to 40 years. Two main themes and eight subthemes emerged from the data (table 1). The facilitating factors and barriers were summarised from narrative passages based on participants' responses. Their quotes were coded and presented in italics. 


\section{Table 1. Facilitating Factors and Barriers to Nurses' Work Performance}

\begin{tabular}{ll}
\hline Themes & Subthemes \\
\hline Facilitators factors & Education and specialty training \\
& Good working environment \\
& Good interpersonal relationships among staff \\
& Effective leadership and management \\
& Self-perceived knowledge and skills insufficiencies \\
Barriers & Moral distress \\
& Logistical concerns \\
& Poor working conditions
\end{tabular}

\section{Theme 1. Facilitating Factors That Improve Nurses' Work Performance}

\section{Education and speciality training}

One of the main facilitating factors that increased work performance in the HD settings included education and speciality training as reported by nearly all nurses interviewed. From their point of view, they had received different work-related trainings that mainly focused on the practical aspects of HD machines, and those trainings highly assisted them in their work performance. Two nurses described their experiences:

Factors that have influenced my job primarily have been training; we did training on connecting and disconnecting HD machines! Connecting patients who have fistulas, then anastomosis for artery and venous, where we can pick the needle. That is a good factor influencing my job (P201). You know with HD, a patient might die in two seconds. You are not able to react well, and the patient is going. I think what it has helped me the most is the Basic Life Support (BLS) course we normally do every year (P202).

\section{Good working environment}

A good working environment was a facilitating factor that increased work performance, and included motivation from colleagues and supervisors, good remuneration, and availability of material and human resources. Two participants explained:

There are many factors, but the first one is the environment that we are working in and having all the necessary items and equipment for doing dialysis, to care for patients on HD. Motivation from supervisors and others helps us to feel well while doing HD and caring for those patients who have a chronic disease. Sometimes it is difficult, but since we are used to working with these patients, we know how to deal with them (P203). They adjust the salary based on job performance. That means that if you have the best salary, it encourages you to perform well. If you perform well, they will give you a lot of money (P401).

\section{Good staff interpersonal relationships}

Most participants emphasised the importance of good interpersonal relationships and good communication practices. A good team spirit increased their work performance, as HD settings required staff to work collaboratively in order to provide quality care. Two participants explained what happened on the HD unit:

If I have a problem, I can call my colleagues or physician, and be can belp me resolve it. It is the team working together that influence me in this unit (P201). We have a good relationship with nurses, even technicians, and the engineer. If we have a problem with the machine, the engineer calls everyone together to show the problem, so next time when we see it we will know what it is about (P402).

\section{Effective leadership and management}

Participants revealed that their work performance was facilitated by the way the management was organised. They emphasised on managerial support to resolve HD problems, availability of policies and procedures, and feedback at meetings and performance appraisals. Several participants recounted:

The unit manager is the head of this unit, and he can organise all the activities for the day or week. If we do not have the unit manager, we will not achieve our objectives. The unit manager helps with team leading, which I think helped us to work better (P201). We have policies and procedures to belp prevent errors or go beyond what is supposed to be done. So they help us to perform well and do what is correct (P203). Every three months there is performance evaluation. When there is something to improve on, according to the stipulated items on the appraisal, I do it. This is very important as I try my best to do well to get high marks (P02). Here we have monthly meetings with the unit manager face-to-face, and you find out what you are doing correctly and what you are doing negatively. Then you make sure you do things correctly (P03). 


\section{Theme 2. Barriers To Improving Nurses' Work Performance}

\section{Self-perceived knowledge and skills insufficiencies}

The participants voiced concern that their educational level and training was insufficient to work on the HD units and therefore a barrier. In particular, they thought they lacked skills to provide emotional support with HD patients. Nearly all expressed a perception of low competence, and hence a negative impact on work performance. Three participants described the situation:

The first one is a lack of training. It is the one factor that can lead to nurses performing poorly on our unit. For example, I think. here all nurses are not trained in the HD unit. For me, I think. that it is a barrier for everyone to perform unit responsibilities poorly (P201). My level of education is not sufficient. numun!! According to the level of education for this HD unit, I do not have sufficient education to perform my assigned activities. I am under the level that I am supposed to have in order to work on this unit. There is another level that I could have to belp me gain more knowledge (P201). We do not have enough clinical competencies in HD, because our level of education is general nursing. We bave no speciality and not enough information about monitoring patients under HD machine (P01).

\section{Moral distress}

Many factors that can be identified as moral distress, related to poor work performance. Moral distress is when the nurse knows what to do, but is not allowed to do it, in other words, the nurse feels powerless. In this regard, considering the high expense of HD treatment and patients' limited financial means to pay for the HD session, the situation caused moral distress. Sometimes nurses were ready to care for their patients, but they were warned not to connect the patients without payment. This affected the nurses, as there was more of a focus on the financial aspect than the patient's needs. One participant shared the following dilemma:

Sometimes the patients have no money to pay, but we have the material, we have time, we have everything that we need to do $i$, but the patient has no money. We have an obligation from our director that we should never, never, never put a patient under the machine without the money. That's a problem for nurses (P01).

Some patients have travelled far for treatment, and the actual HD session takes several hours. It is sometimes challenging to put a patient under the machine if they have not eaten, which results in either the nurse decided not to connect the patient or to take the risk to connect them. A participant explains another dilemma:

Another problem is that some patients are very poor and have no money to buy sometbing to eat. It is tough to put a patient under the machine without eating, and the hospital has no capacity to give food to the patients (P203).
Inadequate staffing clearly affects the performance of nurses in HD settings. An insufficient number of nurses to match the high number of patients in need of HD treatment resulted in a tight schedule and the nurses working extra long hours. One nurse explains it this way:

You see the number of patients compared to the number of nurses. If someone is tired you can't say, I am tired because even if you are tired, you are supposed to work. That is also the challenge, the number of patients always affects us (P401).

Participants reported what they called, "mind management" in which the patient's next-of-kin wanted to be with their patients, and this was associated with many health risks, such as HD catheter infections. When told to stay out of the room they refused, and this has become very difficult for nurses to manage. One nurse explained it this way:

If I say mind management, you know as Africans we like to see our patients and we like to have the next-of-kin nearby the patients. When you tell the next-of-kin, or patients guardians, that they are not allowed to enter the unit, they do not understand. What you want to say is that the hemodialysis catheters are open to blood and even the air has some bacteria circulating. They think that you are preventing them from seeing the patients, so I always give some explanations to those people and slowly, slowly we are getting to the point (P404).

\section{Logistical issues}

Organizational barriers, which were characterised as the physical location, or the flow of the things on the unit, created problems for some staff, patients, and families on the HD units. The HD units were situated far away outside the hospital, while others were far from the intensive care unit (ICU). Not having a convenient location and coordination made it difficult for the movement to and from the HD unit, even for the HD assigned engineer. The logistical issues diverted nurses from their core focus, that of patient care. It had a negative impact on work performance and the patient's health outcome, as one participant recalled:

We are very far from ICU. When we have a very ill patient, a patient who must go in the ICU, or a patient returning from the ICU, the mobilisation is very difficult. When we need a resuscitator or when we need to resuscitate someone, sometimes it takes a long time because they are far from us (P101).

The location of the HD unit might also have contributed to the lack of support staff when nurses needed them. One participant described it this way.

Another problem is the collaboration with technicians... as they have their responsibilities in other services. The main problem is when we have informed them to close the water tank, and then when we come back to work, we find there is no water to use. It makes us very nervous, as we would like to work 
early so that we finish early, but what happens is that we are obliged to wait for sufficient water, and we end up going home very late (P101).

Logistical issues also address infection control on the HD unit as the layout affects the flow of personnel and can help safeguard against infections. Even the flow of visitors to the HD unit made a difference to infection control and altered the way care is given to patients, as one nurse reported:

To make the HD better and to have fewer infections, we are setting many procedures or protocols. Many people think that we are stressing about it, we are stressing, but they don't know the impact of changes, such as when we want to connect the catheters. We are giving them masks, and then they say: $A b ! N o$ ! We went to other hospitals and they are not giving facemasks. So the question, why are you giving these facemasks? And then we explain to them (P404).

\section{Poor working conditions}

Poor working conditions, not covered in other barrier subthemes, included stagnant salary, lack of HD documentation, and poor communication and collaboration. Poor communication was manifested between nursing channels, authorities, and other services. One nurse's voice resounded for all her colleagues:

If there is a meeting for nurses held in the hospital, and we don't attend as we are taking care of patients, we do not know what was discussed. They may have discussed accomplishments for tasks well done, and they may have given warnings. There is no communication with us,we arrive at work every morning, and we occupy our unit. I don't know how it is on other services. It is a problem that may impact us because if there is communication about a concern and we don't know, it's a problem. We don't know how the authorities look at us; I don't know how to explain it. It is a terrible thing (P302).

\section{Discussion}

The findings from the purposive sample of 12 registered nurses working on HD units supported what was already known about nurses' work performance in other countries, yet brought to light what we did not know about nurses working in Rwandan HD units. Two of our facilitating factors and two of our barriers were juxtaposed, meaning they represented two different viewpoints: Education and specialty training was a facilitating factor, and Self-perceived knowledge and skills insufficiencies were considered barriers. Likewise, a Good work environment was seen as a facilitating factor, and Poor work environment a barrier.

For some nurses, their level of education facilitated their HD work experience, while others had a lower level of education and limited competency and performance with HD tasks. The majority of nurses had an advanced diploma in nursing, whereas a few had a bachelor's degree. In this end, nurses thought that their level was not sufficient to perform HD activities and they needed to upgrade. Participants emphasised the importance of training as it facilitated their work performance to care for HD patients. Similarly, a study in Iran, where nurses who received specialised training in dialysis devices, acquired additional HD skills and the capability to manage complications.[14] In contrast, a study in Ethiopia reported limited staff development programs,[15] and in Malaysia higher education had no impact on the quality of care and patient safety.[16]

Nurses perceived a lack of theoretical knowledge and practice gaps in HD skills, in particular, the provision of emotional support needed for CKD patients. Counselling skills are vitally important on the HD unit, as many ESRD patients are depressed due to the permanent loss of kidney function,[17] and lack of appropriate training equates to poor nursing care.[18] Therefore, appropriate training could improve work performance to better support HD patients.

Hemodialysis is a complicated practice that runs the risk of associated errors and harm to the patient.[19] The HD centre is a specialised field with technology that commands highly experienced nurses, preciseness in a detailed work environment, and awareness of the patient's dialysis treatment and potential complications. [17] Thus, HD nurses should be able to recognise early warning signs, screen patients, and prevent HDassociated complications in order for patients to have a longer life. Nurses in the HD units need to upgrade their level of education and speciality training to meet the needs of patients and improve outcomes.

Nurses revealed that good interpersonal relationships among staff improved their work performance. They focused on the importance of good communication with colleagues and patients, relationships, and team decisionmaking. Collaboration among colleagues increased opportunities to share knowledge and information of the patients' condition, which improved the quality care and patients' outcomes, similar to a study in Namibia. [11] In addition, Dutch nurses increased professional collaboration by interacting in a complementary way, having mutual respect, and solving problems as soon as they appeared.[18] This facilitating factor in some HD units in Rwanda should be strengthened.

Nurses who practiced in a good working environment were more likely to have adequate resources (human and materials), satisfaction with work and remuneration, and motivation from supervisors and others. The World Health Organization (WHO) defines working conditions as the combination of compensation, non-financial incentives and workplace safety.[20] Positive Practice Environments are settings that support excellence and a decent work environment: 
"They strive to ensure the health, safety and personal well-being of staff, support quality patient care and improve the motivation, productivity and performance of individuals and organisations.[21] Therefore, it is important to strengthen the HD work environment to ensure sustainability of good performance of nurses, and impact the quality and efficiency of healthcare.[17]

In contrast, a poor work environment had reduced resources, including $\mathrm{HD}$ machines and related documentation. In a study in Namibia, the lack of adequate resources such as materials, equipment, and space lead to poor performance.[10] The same study[10] found, that insufficient equipment and poor remuneration lead to poor performance of nurses. Effective nursing leadership and management practices could organise adequate resources to improve the work environment, which in turn would improve patient care and decrease errors.

Participants emphasised how effective leadership and management enhanced their work performance. Nurses highlighted the role of the team leader who organised the work for the staff nurses, which helped them meet their patient care goals. The staff developed leadership and clinical practice skills that positively influenced their performance. This was a strong area for some HD units in Rwanda that has been sustained. In contrast, a study in Namibia revealed some nurse managers lacked the necessary skills to direct staff training.[11]

Obtaining feedback helped nurses improve their work performance, though some said they did not receive feedback, and others said they were not evaluated, such as the two Namibia studies.[10,11] It is essential for unit managers to provide a timely appraisal on a regular basis to maximise work performance. Equally important as a facilitating factor, was managerial support characterised as a willingness of the authorities to solve HD problems. Though some appreciated the support, others did not. Authors of an Australian study, indicated the quality of nursing care was under the influence of the head nurse, hospital or health centre manager, and nephrologist. [8] In a study by Kamati, lack of managerial support affected job performance,[10] and this is a problem that needs to be rectified for the smooth running of HD units.

Moral distress is defined as "knowing what to do in an ethical situation, but not being allowed to do it,[20] such as the situation involving nurses who had the time and the will to treat the patient, but could not as they lacked sufficient money to pay for their HD treatment or food. Other situations causing moral distress among nurses included a large number of patients to care for on the HD units, overtime hours, lack of break time and thus fatigability, which understandably affected the nurses' work performance. Levels of psychological fatigue were actually greater than physical fatigue, and longer hours were related to more physical and total fatigue levels.[23] The American Association of Critical Care Nurses published the "4 A's to Rising Above Moral Distress" to assist nurses in identifying and responding to moral distress.[22] The one "A's" are:

\section{Ask (am I suffering?) \\ Affirm (commitment to self-care) \\ Assess (the source) \\ Act (to preserve your integrity and authenticity)}

The 4A's are designed for nurses to help reduce frustrations related to situations and give them moral courage. Team members can work together in a collaborative way toward developing a "collective moral courage muscle",[20] and in so doing become better advocates for the nursing profession and patients.

A study of 'inter-shift fatigue,' defined as inadequate recovery time between shifts, resulted in significant fatigue of the nursing staff,[24] and similarly linked to poor nursing care.[8-11,14] When there is fewer staff, those remaining at work have an increased workload and inadequate recovery time between shifts. In Rwanda, few nurses live near hospitals, and therefore most have to travel long distances by public transport early in the morning or in the evening. Some may leave home before their children are awake, and arrive home after the children have gone to bed. Authorities need to react to this continuing problem in order to enhance the nurses' working conditions and patient safety.

Nurses reported that HD centres were situated far from the intensive care unit (ICU) or outside the hospital, which affected their performance and patients' outcomes. This factor understandably hindered patient transportation to and from the ICU and/or HD units. Furthermore, there were delays in pharmacy medications, laboratory results, and resuscitation of critical patients. A request to adapt the infrastructure to facilitate patient care and safety had been initiated with authorities. The layout of hospital units and patient rooms could alter the nurses' capability and patient safety.[25]

\section{Limitations}

This was a small qualitative study so cannot be generalised to other work units or other parts of Rwanda. The viewpoints are only related to a portion of the registered nurses working in Rwandan HD units.

\section{Conclusion}

The findings revealed facilitating factors and barriers to nurses' work performance with hemodialysis patients in Rwanda. Nurses at these study sites, in conjunction with faculty in the Master's Nephrology Track at the University of Rwanda, could become change agents and begin to increase facilitating factors and decrease barriers. Continual improvement in facilitating factors is needed to keep nurses performing well. 


\section{Acknowledgements}

We acknowledge all nurses that participated in the study. We are also thankful to Dr Pamela Meharry for the series of writing retreats that helped enrich this paper.

This article is published open access under the Creative Commons Attribution-NonCommercial NoDerivatives (CC BY-NC-ND 4.0). People can copy and redistribute the article only for noncommercial purposes and as long as they give appropriate credit to the authors. They cannot distribute any modified material obtained by remixing, transforming or building upon this article. See https:// creativecommons.org/licenses/by-nc-nd/4.0/

\section{References}

1. Wachukwu CM, Chimezie P, Chioma E, et al. Prevalence of risk factors for chronic kidney disease among adults in a university community in southern Nigeria. Pan Afr Med J 2016; 21: 1-6.

2. Abbasi MA, Chertow GM, Hall YN. End-stage renal disease. BMJ Clin Evid 2010; 2010: 1-16.

3. Mukakarangwa MC, Chironda G, Bhengu B, et al. Adherence to Hemodialysis and Associated Factors among End Stage Renal Disease Patients at Selected Nephrology Units in Rwanda: A Descriptive CrossSectional Study. Hindawi Nurs Res Pract 2018; 2018: 8.

4. Abo HI, Mohga E, Mohamed S. Effect of an Educational Program on Adherence to Therapeutic Regimen among Chronic Kidney Disease Stage5 ( CKD5 ) Patients under Maintenance Hemodialysis. 2015; 6: 21-34.

5. Naalweh KS, Barakat MA, Sweileh MW, et al. Treatment adherence and perception in patients on maintenance hemodialysis : a cross - sectional study from Palestine. 2017; 1-9.

6. Igiraneza $G$, Ndayishimiye B, Nkeshimana M, et al. Clinical Profile and Outcome of Patients with Acute Kidney Injury Requiring Hemodialysis : Two Years 'Experience at a Tertiary Hospital in Rwanda. Hindawi BioMed Res Int 2018; $2018: 7$.

7. Bennett PN. Technological Intimacy in Haemodialysis Nursing. Nurs Inq 2011; 18: 247-252.

8. Negussie N. An analysis of factors affecting performance of nurses in public hospitals and health centres. Addis Ababa University School, 2010.

9. Ahida Saleem T, Sayej S. Selected Organizational Factors Affecting Performance of Professional Nurses in North West Bank Governmental Hospitals. J Educ Pract 2015; 6: 100-111.

10. Kamati SK. An Evaluation of the Factors Influencing the Performance of Registered Nurses at the National Referral Hospital in Namibia. Aust J Bus Manag Res 2014; 4: 47-62.

11. Awases MH. Factors affecting the performance of professional nurses in Namibia. ResearchGate 2013; 36: 331.
12. Vicent NA. Ensuring the quality of the findings of qualitative research : looking at trustworthiness criteria. J Emerg Trends Educ Res Policy Stud 2015; 5: 272-281.

13. Clarke V, Braun V. Teaching thematic analysis: Overcoming challenges and developing strategies for effective learning. Psychologist 2013; 26: 120-123.

14. Nobahar M, Tamadon MR. Barriers to and facilitators of care for hemodialysis patients; a qualitative study. J Ren Inj Prev 2016; 5: 39-44.

15. Negussie N. An Analytsis of Factors Affecting the Performance of Nurses in Public Hospitals and Health centres in Addis Abbaba. Addis Abbaba University, 2010.

16. Rahman HA, Jarrar M, Don MS. Nurse Level of Education, Quality of Care and Patient Safety in the Medical and Surgical Wards in Malaysian Private Hospitals : A Cross-Sectional Study. Glob J Health Sci 2015; 7: 331-337.

17. Borzou SR, Anosheh M, Mohammadi E, et al. Patients' Perception of Comfort Facilitators During Hemodialysis Procedure: a Qualitative study. Iran Red Crescent Med J 2014; 16: 19055.

18. Kieft RA, de Brouwer BBJM, Francke AL, et al. How nurses and their work environment affect patient experiences of the quality of care: a qualitative study. BMC Health Serv Res 2014; 14: 249.

19. Benjamin D.Bray, Wendy Metcalfe. Safety in HD Editorial Comment Improving patient safety in haemodialysis. Clin Kidney Jounal 2015; 8: 1-3.

20. J Burton. WHO Healthy Workplace Framework and Model: Background and Supporting Literature and Practice, https://www.who.int/occupational_ health/healthy_workplace_framework.pdf (2010).

21. International Council of Nurses. Positive Practice Environments. iInternational Centre for Human Resources in Nursing 2007; 5.

22. Savel RH, Munro CL. Moral D istress, Moral C ourage. AJCC Am J Crit CARE 2015; 24: 276-279.

23. Barker LM, Nussbaum MA. Fatigue, performance and the work environment: A survey of registered nurses. J Adv Nurs 2011; 67: 1370-1382.

24. Geiger-Brown J, Rogers VE, Trinkoff AM, et al. Sleep, sleepiness, fatigue, and performance of 12-hour-shift nurses. Chronobiol Int 2012; 29: 211-219.

25. R.DeLucia P, E.oTT, Palmieri PA. Performance in Nursing. In: Reviews of Human Factors and Ergonomics. 2009, pp. 1-40. 